\title{
Seismic Monitoring in Zhezkazgan Mines — Case Study of a Strong Seismic Event Forecasting
}

\author{
V.I. German Kazakhmys Co. Ltd, Kazakhstan \\ V.A. Mansurov Kazakhmys Co. Ltd, Kazakhstan \\ P.A. Boiko Siberian Aerospace State University, Russia
}

\begin{abstract}
The seismic monitoring program on Zhezkazgan mines and its features are described. Physical concepts of strong seismic event forecasting based on laboratory experiments and earthquake seismology investigations are considered. High concentration of events, seismic gaps and seismicity migration can be used as precursors of strong seismic events. A parameter, which characterises the possibility of weak events interacting and forming a strong seismic event is introduced. The case study of a strong event with radiated energy $10^{7.11} \mathrm{~J}$ which was forecast in real time mode by analysing only seismic data is considered.
\end{abstract}

\section{Introduction}

The room-and-pillar method is used in Zhezkazgan copper mines. Typical depth of mining is $140-300 \mathrm{~m}$. After many decades of mining the overall extraction volume has reached about 180 million $\mathrm{m}^{3}$, which is supported by tens of thousands of rib and inter cavern pillars. An increasing number of destroyed pillars, leading to an increasing number of collapses is contributing to a general degrading of geomechanical conditions caused by an increasing of number of excavated beds and decrease of support for the massif area. Since the 1990s qualitative changes of geomechanical conditions have taken place: some local unstable areas with destroyed pillars have started to unite and form extensive unstable regions and collapses. These processes are accompanied by strong seismic events and air blows in excavations.

\section{Monitoring on Zhezkazgan mines}

To provide safety of mining by means of rock pressure control a special geomechnical service was organised in 1995. The main task of this service is monitoring of excavations, overburden rock, and surface movements. The basic methods of monitoring are:

- Visual inspection of excavations and pillars.

- Geodetic surveying of the ground surface along profiles to monitor subsidence induced by mining.

- Seismic monitoring of the mines.

The main advantages of seismic monitoring is that it provides information on spacious areas covering large volumes (including sites, where visual inspection is not possible) in real time mode. A seismic network registers seismic events - elastic waves, generated in rock mass in a failure moment (crack occurrence moment). Processing of recorded data allows the determination of the following basic parameters of seismic events: location, where failure of rock mass occurred; radiated energy; linear size of the crack/defect corresponded to the event.

The first seismic system was installed in Zhezkazgan mines in 1991. It estimated the amount of seismic activity, but without location of events. To solve this problem in 1997 a second system was put into operation. It had 8 sensors (sites) installed on the surface and this network registers all events with radiated energy more then $E_{\text {min }}=10^{3.6} \mathrm{~J}$ (rough relation between seismic energy $E$ and local magnitude $M$ is $M=0.6 \log _{10} E-0.2$ ) without omission for all area of mines. The seismic catalogue started to be formed from this time. However, the low sensitivity of the system and lack of information has resulted in unpredictability of strong events (failures and collapses). 
To improve accuracy and sensitivity of events location a third seismic system SDSC was put into operation in 2000. It was produced by Elgeo Ltd (Kazakhstan) and had 12 sensors and $E_{\min }$ was reduced to $10^{3.1} \mathrm{~J}$. In 2002 the number of sensors of this system was increased to 20 , that has led to a further decrease of $E_{\min }$ to $10^{2.6} \mathrm{~J}$. Until now this system has been used as a basic system of seismic monitoring.

Processing of seismograms recorded by the seismic system has enabled the creation of a catalogue, which includes information about more than 4700 events. The distribution of seismic events with energy more then $10^{3.6} \mathrm{~J}$ recorded from 01.01.1997 to 01.06.2006 is shown on Figure 1. Each event is shown as a circle with diameter proportional to cube root of radiated energy. Solid line contours corresponds to areas with significant movements of surface caused by collapses. Significant parts of seismic events are concerned with these areas. Some of these areas formed prior to seismic system installation and are no longer active. According to the analysis of sizes of aftershock zones (zones with high seismic activities directly after collapse) the relation between linear size of failure/crack in rock mass $L$ and radiated energy $E$ for Zhezkazgan mines is:

$$
\log _{10} L(m)=1 / 3 \cdot \log _{10} E(J)-0.3 .
$$

On Figure 1 seismic system sites are shown as triangles and some shafts as squares.

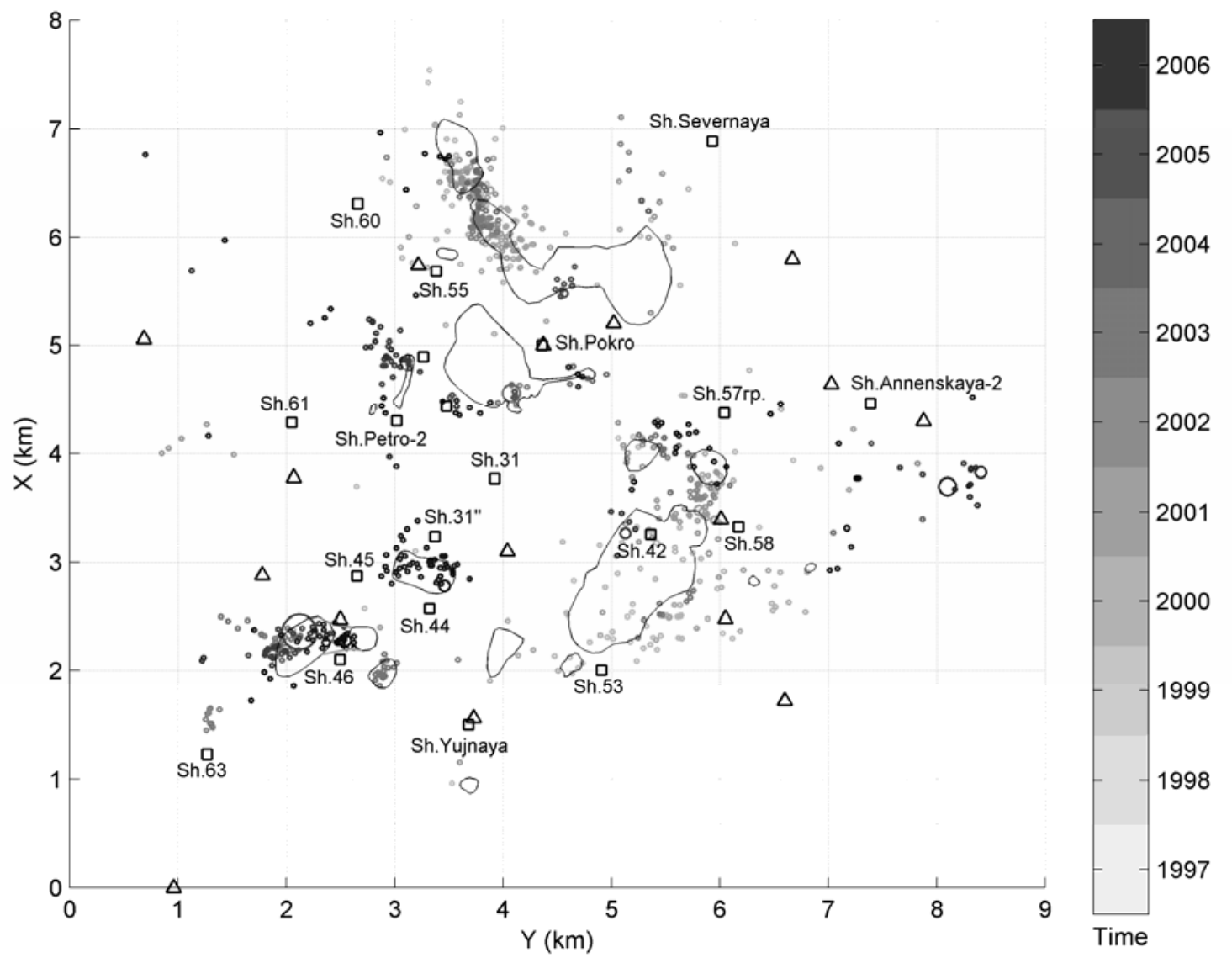

Figure 1 Induced seismicity on Zhezkazgan mines

\section{Physical principles of strong events forecasting}

The procedure of collapse forecasting consists of two basic steps: delineation of areas with high danger of collapses and improvement of the considered area current state estimation with estimation of time to failure.

Experience of Zhezkazgan mines exploitation shows that the conditions for a collapse develop over a long time (years, months), but that the collapse is initiated over a very short time interval (some days) with high intensity. Hence, to forecast collapse the analysis of temporal and spatial (spatial is more important) distribution of seismic events is necessary. 
The basis for forecasting strong seismic events (they can correspond to collapses, rockbursts etc) is rock failure physical concepts, which were established as a result of laboratory experiments and earthquake seismology investigations (Zhurkov, 1965; Locner et al., 1992; Mansurov, 1993; Mogi, 1985; Ponomarev et al., 1997):

- Failure is a process, which is developed in space and time on different scale levels.

- Each strong seismic event corresponds to large scale failure of rock mass prepared by smaller events.

The concentration criteron of solids fracture (Kuksenko et al., 1996) is used as a basic principle to forecast collapses on Zhezkazgan mines. It is based on the analysis of spatial distribution of seismic events and takes into account the scale of each event.

On Zhezkazgan mines the modified concentration criterion is used (German and Mansurov, 2002). The rule of dangerous areas delineation can be easily demonstrated graphically (see Figure 2). Let's associate each event with a sphere having diameter equal $L \cdot \kappa_{*}\left(\kappa_{*}\right.$ is a constant), which roughly corresponds to area of event influence. An intersection of these areas for some events means that potentially they can interact with each other with joining and formation event of the next scale level. The group of such events should be considered as potentially high dangerous. They can be a cause for strong seismic event arising. To estimate hazard of cracks joining, the dimensionless parameter $\kappa$ was introduced (German and Mansurov, 2002). $\kappa$ is the ratio of distance between centers of cracks (between hypocenters of corresponded events) to their average length. This parameter is calculated for each pair of seismic events with indexes $i$ and $j$ :

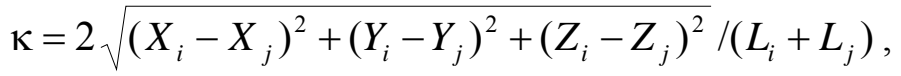

Where:

$X, Y, Z$ - are coordinates of recorded seismic events, caused by crack occurrence with linear size $L$, which is calculated by formula (1) from radiated energy E. Decreasing of parameter $\kappa$ (increasing of cracks/defects concentration) down to threshold value $\kappa_{*}$ leads to occurrence of crack of the next scale level, which can initiate collapse. The theoretical value for $\kappa_{*}$ is 3 (Kuksenko et al., 1996), but for real conditions $\kappa_{*} \approx 5 \ldots 10$.

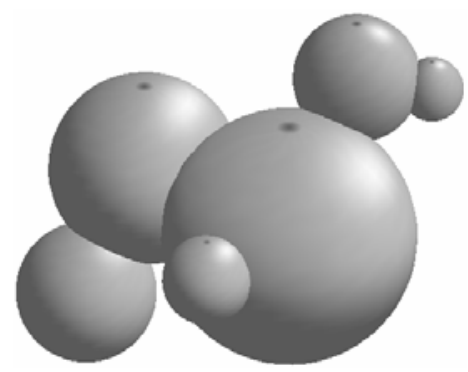

Figure 2 Formation of a dangerous area from areas of influence of seismic events

To delineate areas with high hazard of collapse, seismic events for previous years are back analyzed. For calculation of the parameter $\kappa$, events with radiated energy $E>E_{\max }$ are considered as events with $E=E_{\max }$. This avoids excessive decreasing of the join parameter $\kappa$ in the area of strong seismic event. As a rule $E_{\max } \approx 100 E_{\min } \ldots 1000 E_{\min }$ (German and Mansurov, 2002). Chains of events with $\kappa \leq \kappa_{*}$ united to areas corresponded to high potential for collapse. Analysis of seismic activity in Zhezkazgan mines allows us to establish that zones with $\kappa_{*}=8$ corresponds to potentially dangerous areas and with $\kappa_{*}=5-$ to critical (unstable) ones or to destroyed ones. Thus for Zhezkazgan mines, with its grey sandstones and red rocks (fine grained sandstones, siltstones and claystones) the threshold value of join parameter is $\kappa_{*} \approx 5 \ldots 8$.

Each strong seismic event is preceeded by smaller events with radiated energy that can be down 3 decimal orders less energy of the corresponding strong event (German and Mansurov, 2002). Therefore to forecast strong events with radiated energy $E$ the seismic network should reliably register all events with radiated energy more then $E / 1000$. 
Moreover analysis of expression (2) shows that to guarantee that error of parameter $\kappa$ determination will not exceed $50 \%$ it is necessary: to determine coordinates $X, Y, Z$ with an error not exceeding double size of the crack $L$ (it is about $10 \mathrm{~m}$ for weak events); to determine radiated seismic energy $E$, which is used for calculation of $L$, with error, which does not exceed $300 \%$.

The seismic monitoring systems currently in use in the Zhezkazgan mine does not allow determination of these parameters with such precision in all cases. This reduces the effectiveness of the parameter $\kappa$ application. This problem should be solved after final attenuation of a new seismic system (ISS International Ltd), which was installed in 2007.

Additional important precursors of strong seismic events are "seismic gaps" of the first and second type (areas with anomalous low seismic activity) and migration of seismicity (Mogi, 1985). The seismic gap of the first type is an inactive area, which is surrounded by areas with strong seismic events (with collapses). In this case it can be a barrier between active areas and be under high stress. The failure of this area can leads to strong seismic events occurrence accompanied by joining of adjacent active areas.

Redistribution of stress caused by collapse or failure development can be a reason for seismic event migration with collapse of the correspondng area. Determination of such trends can lead to forecasting the location of future collapse.

A seismic gap of the second type indicates an area with temporary lack of events. It is necessary to accumulate energy for final failure of this area. Often such an area is delineated by active areas which form a "circular structure", which shows the contour of the future collapse.

\section{Case study}

Let us consider the forecasting of one of the strongest event, recorded on Zhezkazgan mines. The event occurred on $17^{\text {th }}$ November 2005 and had a radiated energy $10^{7.11} \mathrm{~J}$. The forecasting was realised in real time mode, and only seismic monitoring information was used.

The room-and-pillar method for 7 overlapped mined levels was used the area affected. This area is characterised by a large number of unstable and caving rooms. This led to the formation of two collapses with significant surface movements (solid contours on Figures 3 and 4). The left/west collapse had started to form in 1979 and was significantly developed in 2000, 2003 and 2005. Each development was accompanied by increasing seismic activity. The right collapse was formed in 1992. Contours of the collapses at the beginning of 2005 are shown on Figures 3 and 4 by the solid line and further developments are shown by the dotted lines with date indication.

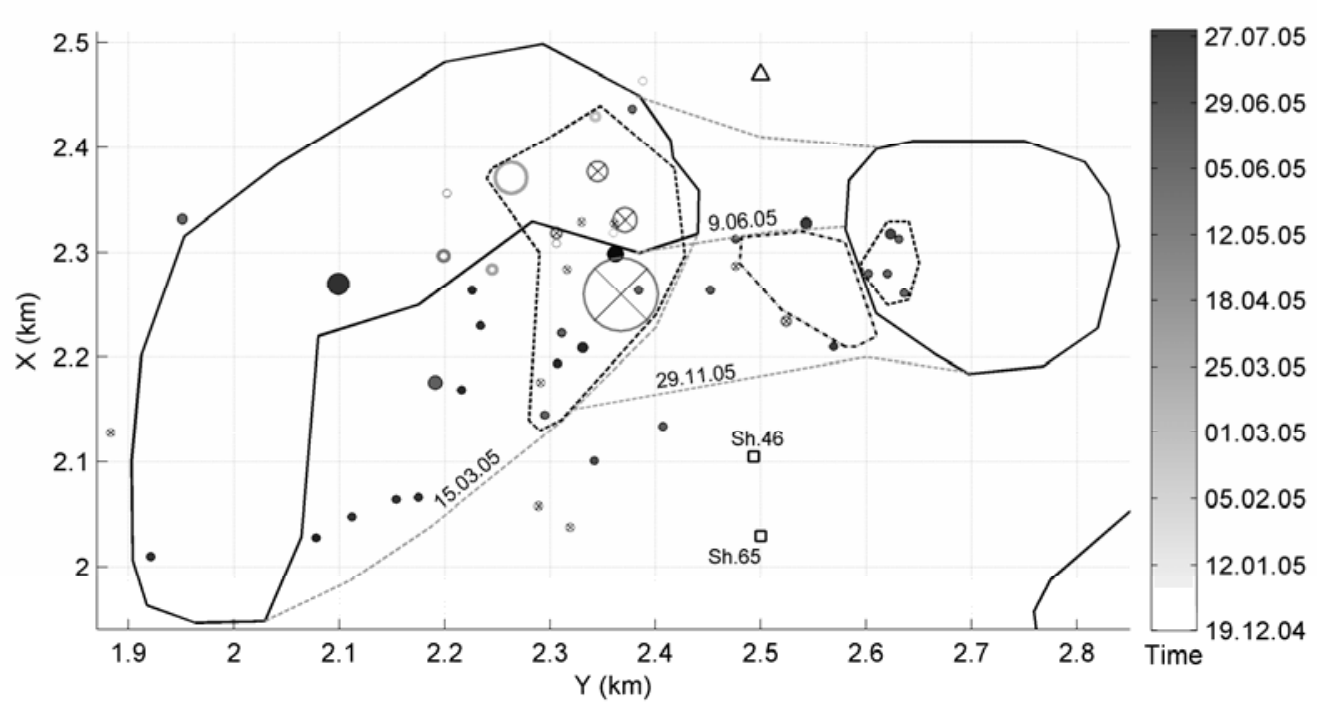

Figure 3 Seismicity prior to the forecasted event occurrence 


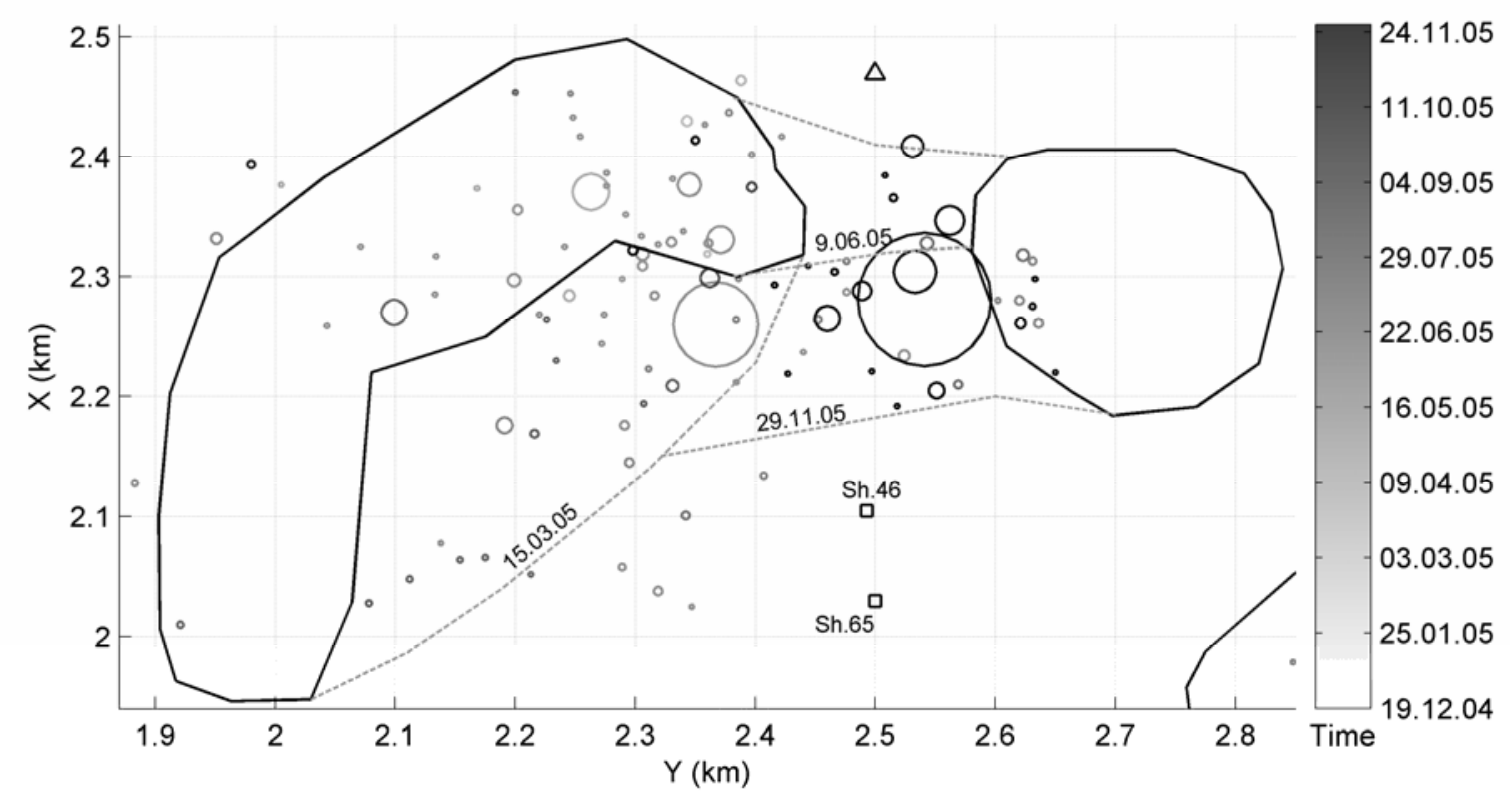

Figure 4 The forecasted strong seismic activity before and after the event

The forecast event occurred between these collapses, when the distance between them reached a value comparable with the linear size of the forecast event. The area between these collapses can be considered as a seismic gap of the first type.

The spatial-temporal and energy/scale distribution of seismicity recorded prior to the strong event occurrence is shown on Figure 3. Only reliably recorded events with radiated energy more then $E_{\min }=10^{2.6} \mathrm{~J}$ are shown. Analysis of Figure 3 demonstrates that the initial moment for preparation of this strong event was 14.03.2005 when another strong event with radiated energy $10^{6.1} \mathrm{~J}$ occurs. During the next five days some new seismic events were recorded. Each of these events is shown on Figure 3 as circles with crosses in the center. Two of these events were between two collapses, where seismic events had not been recorded before (Figure 3). One and a half weeks later seismicity moved to the east with activation of the west part of the left collapse and occurrence of additional events between collapses. These events are shown on Figure 3 as filled circles. Note that seismic activity between collapses in this moment had a circular shape without events in the center (on Figure 3 a dash-dot contour corresponds to this area). The forecast event occurred in this area later and was considered as a gap area of the second type. Moreover, to the left and the right of this gap area are two areas where a high value of parameter $\kappa$ was formed. They correspond to dotted contours on Figure $3, \kappa_{*}=5$, $E_{\max }=100 \cdot E_{\min }=10^{4.6} \mathrm{~J}$. Thus the following conclusion was derived: the formation of a seismic gap of the first type between two collapses is finished. As a result, the forecast of high danger in this area was made and mining was stopped prior to the collapse which occurred at $17^{\text {th }}$ November 2005.

The forecast event is shown on Figure 4 as the circle with maximum diameter. On the same figure all recorded events before and after the collapse are shown. The active stage of the collapse correspondent to the strong events lasting about one week. As a result four rooms collapsed, significant movements occurred at the surface and as a result, led to the uniting of the nearby collapses. The movement areas involve a road and a heating main to Shaft 65 .

\section{Conclusion}

Mine wide seismic systems can be used to monitor the failure processes in the rock mass. The analysis of induced seismicity with application of modern physical concepts allows forecasting of future collapses. Thus it allows a reduction of the risk such events bring in difficult mining and geological conditions. 


\section{References}

German, V.I. and Mansurov, V.A. (2002) Induced seismicity monitoring and procedure of rock-burst focus localization. Journal of Mining Science, Vol. 38 (4), pp. 336-343.

Kuksenko, V.S., Tomilin, N.G., Damaskinskaya, E. and Lockner, D.A. (1996) A two-stage model of fracture of rocks. Pure and Applied Geophysics, Vol. 146 (2), pp. 253-263.

Lockner, D.A., Byerlee, J.D., Kuksenko, V., Ponomarev, A. and Sidorin, A. (1992) Observations of quasistatic fault growth from acoustic emissions. In Fault Mechanics and Transport Properties of Rock. B. Evans and T.F. Wong (eds), Academic Press, London, pp. 3-31.

Mansurov, V.A. (1993) Laboratory Experiments: Their role in the Problem of Rock Burst Prediction. Comprehensive Rock Engineering, Vol. 3, London, Pergamon Press.

Mogi, K. (1985) Earthquake prediction. Academic Press, Tokyo.

Ponomarev, A.V., Zavyalov, A.D., Smirnov, V.B., Lockner, D.A. (1997) Physical modeling of the formation and evolution of seismically active fault zones. Tectonophysics, Vol. 277, pp. 57-81.

Zhurkov, S.N. (1965) Kinetic concept of the strength of solids. International Journal of Fracture Mechanics, Vol. 1, pp. 311-323. 